\title{
Generalized Malcev-Neumann Series Modules with the Beachy-Blair Condition
}

\author{
Mohamed A. Farahat ${ }^{1,2}$ \\ ${ }^{1}$ Department of Mathematics and Statistics, Faculty of Science, Taif University, P.O. Box 888, Al-Hawiyah, Taif 21974, Saudi Arabia \\ ${ }^{2}$ Mathematics Department, Faculty of Science, Al-Azhar University, P.O. Box 11884, Nasr City, Cairo, Egypt
}

Correspondence should be addressed to Mohamed A. Farahat; m_farahat79@yahoo.com

Received 9 January 2015; Revised 9 March 2015; Accepted 10 March 2015

Academic Editor: Andrei V. Kelarev

Copyright (C) 2015 Mohamed A. Farahat. This is an open access article distributed under the Creative Commons Attribution License, which permits unrestricted use, distribution, and reproduction in any medium, provided the original work is properly cited.

We introduce a new class of extension rings called the generalized Malcev-Neumann series ring $R((S ; \sigma ; \tau))$ with coefficients in a ring $R$ and exponents in a strictly ordered monoid $S$ which extends the usual construction of Malcev-Neumann series rings. Ouyang et al. in 2014 introduced the modules with the Beachy-Blair condition as follows: A right $R$-module satisfies the right Beachy-Blair condition if each of its faithful submodules is cofaithful. In this paper, we study the relationship between the right Beachy-Blair condition of a right $R$-module $M_{R}$ and its Malcev-Neumann series module extension $M((S))_{R((S ; \sigma ; \tau))}$.

\section{Introduction}

Throughout this paper $R$ denotes an associative ring with identity; $(S, \cdot, \leqslant)$ is a strictly ordered monoid (i.e., $(S, \leqslant)$ is an ordered monoid satisfying the conditions that if $s<s^{\prime}$, then $s t<s^{\prime} t$ and $t s<t s^{\prime}$ for $\left.s, s^{\prime}, t \in S\right)$. Recall that a subset $X$ of $(S, \leqslant)$ is said to be artinian if every strictly decreasing sequence of elements of $X$ is finite and that $X$ is narrow if every subset of pairwise order-incomparable elements of $X$ is finite. Suppose the two maps $\sigma: S \rightarrow \operatorname{End}(R)$ and $\tau: S \times S \rightarrow U(R)$ (the group of invertible elements of $R)$. Let $A=R((S ; \sigma ; \tau))$ denote the set of all formal sums $f=\sum_{x \in S} a_{x} \bar{x}$ such that $\operatorname{supp}(f)=\left\{x \in S \mid a_{x} \neq 0\right\}$ is an artinian and narrow subset of $S$, with componentwise addition and the multiplication rule is given by

$$
\begin{aligned}
& \left(\sum_{x \in S} a_{x} \bar{x}\right)\left(\sum_{y \in S} b_{y} \bar{y}\right) \\
& =\sum_{z \in S}\left(\sum_{\{(x, y) \mid x y=z\}} a_{x} \sigma_{x}\left(b_{y}\right) \tau(x, y)\right) \bar{z},
\end{aligned}
$$

for each $\sum_{x \in S} a_{x} \bar{x}$ and $\sum_{y \in S} b_{y} \bar{y} \in A$. In order to ensure the associativity, it is necessary to impose two additional conditions on $\sigma$ and $\tau$ : namely, for all $x, y, z \in S$, (i) $\sigma_{x}(\tau(y, z)) \tau(x, y z)=\tau(x, y) \tau(x y, z)$,

(ii) $\sigma_{x} \sigma_{y}=\eta(x, y) \sigma_{x y}$, where $\eta(x, y)$ denotes the automorphism of $R$ defined by

$$
\eta(x, y)(r)=\tau(x, y) r \tau(x, y)^{-1} \quad \forall r \in R .
$$

It is now routine to check that $A=R((S ; \sigma ; \tau))$ is a ring which is called the ring of generalized Malcev-Neumann series. We can assume that the identity element of $A$ is $\overline{1}$; this means that

$$
\sigma_{1}=\operatorname{Id}_{R}, \quad \tau(x, 1)=\tau(1, x)=1 .
$$

In this case $r \mapsto r \overline{1}$ is an embedding of $R$ as a subring into $A$.

For each $f \in A \backslash\{0\}$ we denote by $\pi(f)$ the set of minimal elements of $\operatorname{supp}(f)$. If $(S, \leq)$ is a strictly totally ordered monoid, then $\operatorname{supp}(f)$ is a nonempty well-ordered subset of $S$ and $\pi(f)$ consists of only one element.

Clearly, the above construction generalizes the construction of Malcev-Neumann series rings, in case of $S=G$ (an ordered group), which was introduced independently by Malcev and Neumann (see $[1,2])$.

If the order $\leq$ is the trivial order, then $A=R((S ; \sigma ; \tau))$ is the usual crossed product ring $R[S ; \sigma ; \tau]$. Also, if the monoid $S$ has the trivial order and $\tau$ is trivial, then $A=R((S ; \sigma ; \tau))$ is 
the usual skew monoid ring $R[S ; \sigma]$. However if the monoid $S$ has the trivial order and $\sigma$ is trivial, then $A=R((S ; \sigma ; \tau))$ is the usual twisted monoid ring $R[S ; \tau]$. Finally, if the monoid $S$ has the trivial order and $\sigma$ and $\tau$ are trivial, then $A=R((S ; \sigma ; \tau))$ is the usual monoid ring $R[S]$ (see Sections 3.2 and 3.3 in [3]).

Moreover, if $\alpha$ is a ring endomorphism of $R$, set $S=\mathbb{Z}_{\geq 0}$ endowed with the trivial order. Define $\sigma: S \rightarrow \operatorname{End}(R)$ via $\sigma(x)=\alpha^{x}$ for every $x \in \mathbb{Z}_{\geq 0}$ and $\tau(x, y)=1$ for any $x, y \in \mathbb{Z}$. We have $A=R((S ; \sigma ; \tau))$ is the usual skew polynomial ring $R[x, \alpha]$. However if $\leq$ is the usual order, then $A=R((S ; \sigma ; \tau))$ is the usual skew power series ring $R[[x, \sigma]]$. If $\alpha$ is a ring automorphism of $R, S=\mathbb{Z}$ and $\leq$ is the usual order, then $A=R((S ; \sigma ; \tau))$ is the usual ring of skew Laurent power series $R\left[\left[x, x^{-1}, \alpha\right]\right]$.

At the same time, if we set also $\sigma(s)=\operatorname{Id}_{R} \in \operatorname{End}(R)$ for all $s \in S$, then it is easy to check that polynomial rings, Laurent polynomial rings, formal power series rings, and Laurent power series rings are special cases of $A=R((S ; \sigma ; \tau))$.

If $M_{R}$ is a unitary right $R$-module, then the MalcevNeumann series module $B=M((S))$ is the set of all formal sums $\sum_{x \in S} m_{x} \bar{x}$ with coefficients in $M$ and artinian and narrow supports, with pointwise addition and scalar multiplication rule is defined by

$$
\begin{aligned}
& \left(\sum_{x \in S} m_{x} \bar{x}\right)\left(\sum_{y \in S} a_{y} \bar{y}\right) \\
& =\sum_{z \in S}\left(\sum_{\{(x, y) \mid x y=z\}} m_{x} \sigma_{x}\left(a_{y}\right) \tau(x, y)\right) \bar{z}
\end{aligned}
$$

where $\sum_{x \in S} m_{x} \bar{x} \in B$ and $\sum_{y \in S} a_{y} \bar{y} \in A$. One can easily check that (i) and (ii) ensure that $M((S))$ is a unitary right $A$-module. For each $\varphi \in B \backslash\{0\}$ we denote by $\pi(\varphi)$ the set of minimal elements of $\operatorname{supp}(\varphi)$. If $(S, \leq)$ is a strictly totally ordered monoid, then $\operatorname{supp}(\varphi)$ is a nonempty well-ordered subset of $S$ and $\pi(\varphi)$ consists of only one element.

Recall from Faith [4] that a ring $R$ is called a right zip ring and if the right annihilator $\mathrm{r}_{R}(X)$ of a subset $X \subseteq R$ is zero, then $\mathrm{r}_{R}\left(X_{0}\right)=0$ for a finite subset $X_{0}$ of $X$. Although the concept of zip rings was initiated by Zelmanowitz [5] it was not called so at that time.

Recall from [6] that a right $R$-module $M_{R}$ is called a right $z i p$ module provided that if the right annihilator of a subset $X$ of $M_{R}$ is zero, then there exists a finite subset $X_{0} \subseteq X$ such that $\mathrm{r}_{R}\left(X_{0}\right)=0$.

According to Rodríguez-Jorge [7], a ring $R$ satisfies the right Beachy-Blair condition if its faithful right ideals are cofaithful; that is, if $I$ is a right ideal of $R$ such that $\mathrm{r}_{R}(I)$ vanishes, then $\mathrm{r}_{R}\left(I_{0}\right)=0$ for a finite subset $I_{0}$ of $I$. Clearly, a right zip ring is a right Beachy-Blair ring.

Ouyang et al. in [8] generalized the right Beachy-Blair condition from rings into modules as follows: A right $R$ module $M_{R}$ is called module with the Beachy-Blair condition provided that if the right annihilator of a submodule $N_{R}$ of $M_{R}$ is zero, then there exists a finite subset $N_{0} \subseteq N$ such that $\mathrm{r}_{R}\left(N_{0}\right)=0$.

The main aim of the present paper is to investigate conditions for the Malcev-Neumann series modules $M((S))_{R((S ; \sigma ; \tau))}$ to satisfy the right Beachy-Blair condition. The proofs of our results obtained here are very similar to those obtained by Ouyang et al. in [8] and by Salem et al. in [9].

\section{Generalized Malcev-Neumann Series Modules with the Beachy-Blair Condition}

We start this section with the following notions and definitions.

Let $V$ be a subset of $M_{R}$; then

$V((S))$

$$
=\left\{\varphi=\sum_{x \in S} m_{x} \bar{x} \in B \mid 0 \neq m_{x} \in V, x \in \operatorname{supp}(\varphi)\right\} .
$$

Definition 1. A ring $R$ is called $S$-compatible if, for all $a, b \in R$ and $x \in S, a b=0$ if and only if $a \sigma_{x}(b)=0$.

Definition 2. A right $R$-module $M_{R}$ is called $S$-compatible if, for each $m \in M, a \in R$, and $x \in S$, $m a=0$ if and only if $m \sigma_{x}(a)=0$.

Definition 3. A ring $R$ is called $S$-Armendariz if whenever $f g=0$ implies $a_{x} \sigma_{x}\left(b_{y}\right)=0$ for each $x \in \operatorname{supp}(f)$ and $y \in \operatorname{supp}(g)$, where $f=\sum_{x \in S} a_{x} \bar{x}$ and $g=\sum_{y \in S} b_{y} \bar{y}$ are elements of $A$.

We extend the S-Armendariz concept to modules as follows.

Definition 4. A right $R$-module $M_{R}$ is called $S$-Armendariz if whenever $\varphi f=0$ implies $m_{x} \sigma_{x}\left(a_{y}\right)=0$ for each $x \in$ $\operatorname{supp}(\varphi)$ and $y \in \operatorname{supp}(f)$, where $\varphi=\sum_{x \in S} m_{x} \bar{x} \in B$ and $f=\sum_{y \in S} a_{y} \bar{y} \in A$.

It is clear that $R$ is an $S$-Armendariz ( $S$-compatible) ring if and only if $R_{R}$ is an $S$-Armendariz ( $S$-compatible) module.

For a subset $U$ of $M_{R}$, we define $\mathrm{r}_{A}(U)$ as the set

$$
\mathrm{r}_{A}(U)=\{f \in A \mid(u \overline{1}) f=0 \text { for each } u \in U\} .
$$

Lemma 5. Let $M_{R}$ be a right $R$-module. Then $\mathrm{r}_{A}(U)=$ $\mathrm{r}_{R}(U)((S ; \sigma ; \tau))$, for any subset $U$ of $M_{R}$.

Proof. Let $f=\sum_{s \in S} a_{s} \bar{s} \in \mathrm{r}_{A}(U)$. Then for each $u \in U$ we have $(u \overline{1}) f=0$. Thus

$$
0=(u \overline{1})\left(\sum_{s \in S} a_{s} \bar{s}\right)=\sum_{s \in S} u \sigma_{1}\left(a_{s}\right) \tau(1, s) \bar{s}=\sum_{s \in S} u a_{s} \bar{s},
$$

which implies that $u a_{s}=0$ for each $s \in \operatorname{supp}(f)$. Hence $a_{s} \in \mathrm{r}_{R}(U)$ for each $s \in \operatorname{supp}(f)$. So $f \in \mathrm{r}_{R}(U)((S ; \sigma ; \tau))$ and $\mathrm{r}_{A}(U) \subseteq \mathrm{r}_{R}(U)((S ; \sigma ; \tau))$.

On the other hand, suppose that $f=\sum_{s \in S} a_{s} \bar{s} \in \mathrm{r}_{R}(U)$ $((S ; \sigma ; \tau))$; then $a_{s} \in \mathrm{r}_{R}(U)$ for each $s \in \operatorname{supp}(f)$. Thus $u a_{s}=0$ for each $u \in U$, which implies that $u \sigma_{1}\left(a_{s}\right) \tau(1, s)=0$ for each $u \in U$ and $s \in \operatorname{supp}(f)$. Hence $(u \overline{1}) f=0$ and $f \in \mathrm{r}_{A}(U)$. So $\mathrm{r}_{R}(U)((S ; \sigma ; \tau)) \subseteq \mathrm{r}_{A}(U)$. Therefore $\mathrm{r}_{A}(U)=\mathrm{r}_{R}(U)((S ; \sigma ; \tau))$. 
When $M_{R}=R_{R}$ we have the following consequence of Lemma 5.

Corollary 6. Consider $\mathrm{r}_{A}(U)=\mathrm{r}_{R}(U)((S ; \sigma ; \tau))$, for any subset $U$ of $R$.

Note the following: for $\varphi=\sum_{x \in S} m_{x} \bar{x} \in B$, let $\mathrm{C}_{\varphi}=\left\{m_{x} \mid\right.$ $x \in S\}$ and for a subset $V \subseteq M((S))$, we have $\mathrm{C}_{V}=\mathrm{U}_{\varphi \in V} \mathrm{C} \varphi$.

Lemma 7. Let $M_{R}$ be an S-compatible and S-Armendariz Rmodule. Then

$$
\mathrm{r}_{A}(V)=\mathrm{r}_{R}\left(\mathrm{C}_{V}\right)((S ; \sigma ; \tau))
$$

for any $V \subseteq B$.

Proof. Let $V \subseteq B$ and $T=\mathrm{C}_{V}=\cup_{\varphi \in V} \mathrm{C} \varphi=\cup_{\varphi \in V}\left\{m_{x} \mid x \in S\right\}$. We show that $\mathrm{r}_{A}(V)=\mathrm{r}_{R}(T)((S ; \sigma ; \tau))$ and it is enough to show that $\mathrm{r}_{A}(\varphi)=\mathrm{r}_{R}(C \varphi)((S ; \sigma ; \tau))$ for each $\varphi=\sum_{x \in S} m_{x} \bar{x} \epsilon$ $V$. In fact, let $f=\sum_{y \in S} a_{y} \bar{y} \in \mathrm{r}_{A}(\varphi)$. Then $\varphi f=0$. Since $M_{R}$ is an $S$-Armendariz module, $m_{x} a_{y}=0$ for each $x \in \operatorname{supp}(\varphi)$ and $y \in \operatorname{supp}(f)$. Then $a_{y} \in \mathrm{r}_{R}\left(\mathrm{C}_{\varphi}\right)$ for each $y \in \operatorname{supp}(f)$. Thus $f \in \mathrm{r}_{R}(\mathrm{C} \varphi)((S ; \sigma ; \tau))$ and $\mathrm{r}_{A}(\varphi) \subseteq \mathrm{r}_{R}(C \varphi)((S ; \sigma ; \tau))$. Now, let $f=\sum_{y \in S} a_{y} \bar{y} \in \mathrm{r}_{R}(\mathrm{C} \varphi)((S ; \sigma ; \tau))$. Then $a_{y} \in \mathrm{r}_{R}(\mathrm{C} \varphi)$ for each $y \in \operatorname{supp}(f)$. Hence $m_{x} a_{y}=0$ for each $x \in \operatorname{supp}(\varphi)$ and $y \in \operatorname{supp}(f)$. Since $M_{R}$ is $S$-compatible, it follows that $m_{x} \sigma_{x}\left(a_{y}\right)=0$, which implies that $m_{x} \sigma_{x}\left(a_{y}\right) \tau(x, y)=0$ for each $x \in \operatorname{supp}(\varphi)$ and $y \in \operatorname{supp}(f)$. Consequently

$$
0=\sum_{z \in S}\left(\sum_{\{(x, y) \mid x y=z\}} m_{x} \sigma_{x}\left(a_{y}\right) \tau(x, y)\right) \bar{z}=\varphi f .
$$

So $f \in \mathrm{r}_{A}(\varphi)$ and it follows that $\mathrm{r}_{R}(C \varphi)((S ; \sigma ; \tau)) \subseteq \mathrm{r}_{A}(\varphi)$. So

$$
\begin{aligned}
\mathrm{r}_{A}(V) & =\bigcap_{\varphi \in V} \mathrm{r}_{A}(\varphi)=\bigcap_{\varphi \in V} \mathrm{r}_{R}(\mathrm{C} \varphi)((S ; \sigma ; \tau)) \\
& =\left(\bigcap_{\varphi \in V} \mathrm{r}_{R}(\mathrm{C} \varphi)\right)((S ; \sigma ; \tau)) \\
& =\mathrm{r}_{R}(T)((S ; \sigma ; \tau))=\mathrm{r}_{R}\left(\mathrm{C}_{V}\right)((S ; \sigma ; \tau)) .
\end{aligned}
$$

For a right $R$-module $M_{R}$, we define

$$
\begin{aligned}
\mathrm{r}_{R}\left(2^{M}\right) & =\left\{\mathrm{r}_{R}(U) \mid U \subseteq M\right\}, \\
\mathrm{r}_{A}\left(2^{B}\right) & =\left\{\mathrm{r}_{A}(V) \mid V \subseteq B\right\} .
\end{aligned}
$$

Lemma 5 gives us the map $\Pi: \mathrm{r}_{R}\left(2^{M}\right) \rightarrow \mathrm{r}_{A}\left(2^{B}\right)$ defined by $\Pi(I)=I((S ; \sigma ; \tau))$ for every $I \in \mathrm{r}_{R}\left(2^{M}\right)$. Obviously $\Pi$ is an injective map.

In the following lemma we show that $\Pi$ is a bijective map if and only if $M_{R}$ is $S$-Armendariz.

Lemma 8. Let $M_{R}$ be an S-compatible R-module. The following conditions are equivalent.

(1) $M_{R}$ is an S-Armendariz R-module.
(2) $\Pi: \mathrm{r}_{R}\left(2^{M}\right) \rightarrow \mathrm{r}_{A}\left(2^{B}\right)$ defined by $\Pi(I)=I((S ; \sigma ; \tau))$ is a bijective map.

Proof. (1) $\Rightarrow(2)$.

It is only necessary to show that $\Pi$ is surjective. Let $V \subseteq B$ and $T=\mathrm{C}_{V}$. Since $\Pi\left(\mathrm{r}_{R}(T)\right)=\mathrm{r}_{R}(T)((S ; \sigma ; \tau))$, the proof of this direction follows directly from Lemma 7.

(2) $\Rightarrow(1)$.

Let $f=\sum_{y \in S} a_{y} \bar{y} \in A$ and $\varphi=\sum_{x \in S} m_{x} \bar{x} \in B$ such that $\varphi f=0$. Then $f \in \mathrm{r}_{A}(\varphi)$. By assumption $\mathrm{r}_{A}(\varphi)=T((S ; \sigma ; \tau))$ for some right ideal $T$ of $R$. Hence $f \in T((S ; \sigma ; \tau))$ which implies that $a_{y} \in T \subseteq \mathrm{r}_{A}(\varphi)$ for each $y \in \operatorname{supp}(f)$. So, $\varphi\left(a_{y} \overline{1}\right)=0$ and we have that

$$
0=\left(\sum_{x \in S} m_{x} \bar{x}\right)\left(a_{y} \overline{1}\right)=\sum_{x \in S} m_{x} \sigma_{x}\left(a_{y}\right) \tau(x, 1) \bar{x}
$$

for each $x \in \operatorname{supp}(\varphi)$ and $y \in \operatorname{supp}(f)$. Thus $m_{x} \sigma_{x}\left(a_{y}\right)=$ 0 for each $x \in \operatorname{supp}(\varphi)$ and $y \in \operatorname{supp}(f)$. So, $M_{R}$ is an $S$ Armendariz module.

Recall that a ring is reduced if it has no nonzero nilpotent elements. Reduced rings have been studied for over fortyeight years (see [10]). In 2004, the reduced ring concept was extended to modules by Lee and Zhou [11] as follows: a right $R$-module $M_{R}$ is reduced if, for any $m \in M_{R}$ and any $a \in R$, $m a=0$ implies $m R \cap M a=0$. Clearly, if $M_{R}$ is reduced, then, for all $m \in M_{R}$ and $a \in R, m a=0$ implies $m R a=0$. It is clear that $R$ is a reduced ring if and only if $R_{R}$ is a reduced module.

Now, we are able to prove the main result.

Theorem 9. Let $M_{R}$ be a reduced, S-compatible, and $S$ Armendariz right $R$-module. If $M_{R}$ satisfies the right BeachyBlair condition, then $B_{A}$ satisfies the right Beachy-Blair condition.

Proof. Suppose that a right $R$-module $M_{R}$ satisfies the right Beachy-Blair condition and $J$ is a right $A$-submodule of $B$ such that $\mathrm{r}_{A}(J)=0$.

From Lemma 8, we conclude that $\mathrm{r}_{R}\left(\mathrm{C}_{J}\right)((S ; \sigma ; \tau))=$ $\Pi\left(\mathrm{r}_{R}\left(\mathrm{C}_{J}\right)\right)=\mathrm{r}_{A}(J)=0$. Thus $\mathrm{r}_{R}\left(\mathrm{C}_{J}\right)=0$.

Let $C_{J} R$ denote the right $R$-submodule of $M_{R}$ generated by $\mathrm{C}_{J}$. Since $\mathrm{C}_{J} \subset \mathrm{C}_{J} R$, we have $\mathrm{r}_{R}\left(\mathrm{C}_{J} R\right) \subset \mathrm{r}_{R}\left(\mathrm{C}_{J}\right)=0$. Since $M_{R}$ satisfies the right Beachy-Blair condition, there exists a finite subset

$$
X=\left\{\sum_{i=1}^{n_{t}} q_{i}^{t} r_{i}^{t} \mid q_{i}^{t} \in \mathrm{C}_{J}, r_{i}^{t} \in R, 1 \leq t \leq k\right\} \subset \mathrm{C}_{J} R,
$$

such that $\mathrm{r}_{R}(X)=0$. Let

$$
X_{0}=\left\{q_{1}^{1}, q_{2}^{1}, \ldots, q_{n_{1}}^{1}, q_{1}^{2}, q_{2}^{2}, \ldots, q_{n_{2}}^{2}, q_{1}^{k}, q_{2}^{k}, \ldots, q_{n_{k}}^{k}\right\} .
$$

Then $X_{0}$ is a finite subset of $C_{J}$. Now we will see that $\mathrm{r}_{R}\left(X_{0}\right)=$ 0 . Let $a \in \mathrm{r}_{R}\left(X_{0}\right)$; then $q_{i}^{t} a=0$ for $1 \leq i \leq n_{t}$ and $1 \leq t \leq k$. Since $M_{R}$ is a reduced $R$-module, then $q_{i}^{t} r_{i}^{t} a=0$ for $1 \leq i \leq$ $n_{t}$ and $1 \leq t \leq k$. Then for each $\left(\sum_{i=1}^{n_{t}} q_{i}^{t} r_{i}^{t}\right) \in X$, we have $\left(\sum_{i=1}^{n_{t}} q_{i}^{t} r_{i}^{t}\right) a=0$. Therefore $a \in \mathrm{r}_{R}(X)=0$, and so $\mathrm{r}_{R}\left(X_{0}\right)=0$ is proved. 
For each $q_{i}^{t} \in X_{0}$, there exists an element $\varphi_{i}^{t} \in J$ such that $q_{i}^{t} \in \mathrm{C}_{\varphi_{i}^{t}}$. Let $V$ be a minimal subset of $J$ such that $\varphi_{i}^{t} \in V$ for each $q_{i}^{t} \in X_{0}$; then $V$ is a finite subset of $J$ and $X_{0} \subset \mathrm{C}_{V}$. Thus $\mathrm{r}_{R}\left(\mathrm{C}_{V}\right) \subset \mathrm{r}_{R}\left(X_{0}\right)=0$. Now we show that $\mathrm{r}_{A}(V)=0$. Let the contrary; that is, $\mathrm{r}_{A}(V) \neq 0$, and suppose that $f=\sum_{y \in S} b_{y} \bar{y} \in$ $\mathrm{r}_{A}(V) \backslash\{0\}$; then $\varphi f=0$ for each $\varphi=\sum_{x \in S} a_{x} \bar{x} \in V$. Let $y \in \operatorname{supp}(f)$; since $M_{R}$ is an $S$-Armendariz and $S$-compatible module, we have $a_{x} b_{y}=0$ for all $a_{x} \in \mathrm{C}_{\varphi}$ and each $\varphi \in V$. Hence $b_{y} \in \mathrm{r}_{R}\left(\mathrm{C}_{V}\right)=0$, a contradiction. Hence $\mathrm{r}_{A}(V)=0$ is proved. Thus $B_{A}$ satisfies the right Beachy-Blair condition.

When $M_{R}=R_{R}$ we have the following consequence of Theorem 9 .

Corollary 10. Suppose that $R$ is a reduced, S-compatible, and S-Armendariz ring. If $R$ satisfies the right Beachy-Blair condition, then A satisfies the right Beachy-Blair condition.

\section{Conflict of Interests}

The author declares that there is no conflict of interests regarding the publication of this paper.

\section{Acknowledgment}

The author would like to express deep gratitude to the referee for his/her valuable suggestions which improved the presentation of the paper.

\section{References}

[1] A. Malcev, "On embedding of group algebras in a division algebra," Doklady Akademii Nauk, vol. 60, pp. 1499-1501, 1948 (Russian).

[2] B. H. Neumann, "On ordered division rings", Transactions of the American Mathematical Society, vol. 66, pp. 202-252, 1949.

[3] A. V. Kelarev, Ring Constructions and Applications, World Scientific, River Edge, NJ, USA, 2002.

[4] C. Faith, "Annihilator ideals, associated primes and KaschMcCoy commutative rings," Communications in Algebra, vol. 19, no. 7, pp. 1867-1892, 1991.

[5] J. M. Zelmanowitz, "The finite intersection property on annihilator right ideals," Proceedings of the American Mathematical Society, vol. 57, no. 2, pp. 213-216, 1976.

[6] C. Zhang and J. Chen, "Zip modules," Northeastern Mathematical Journal, vol. 24, pp. 240-256, 2008.

[7] E. Rodríguez-Jorge, "Rings with the Beachy-Blair condition," Journal of Algebra and Its Applications, vol. 11, no. 1, Article ID 1250006, 2012.

[8] L. Ouyang, J. Liu, and Y. Xiang, "Modules with the Beachy-Blair condition," Communications in Algebra, vol. 42, no. 2, pp. 853871, 2014.

[9] R. M. Salem, A. M. Hassanein, and M. A. Farahat, "Mal'cevNeumann series over zip and weak zip rings," Asian-European Journal of Mathematics, vol. 5, no. 4, 2012.

[10] G. Renault, "Anneaux reduits non commutatifs," Journal de Mathématiques Pures et Appliquées, vol. 46, pp. 203-214, 1967.
[11] T.-K. Lee and Y. Zhou, "Reduced modules," in Rings, Modules, Algebras and Abelian Groups, vol. 236 of Lecture Notes in Pure and Applied Mathematics, pp. 365-377, Marcel Dekker, New York, NY, USA, 2004. 


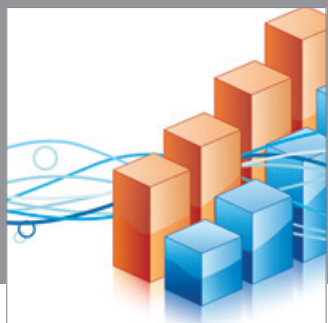

Advances in

Operations Research

mansans

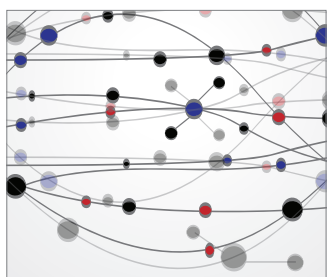

The Scientific World Journal
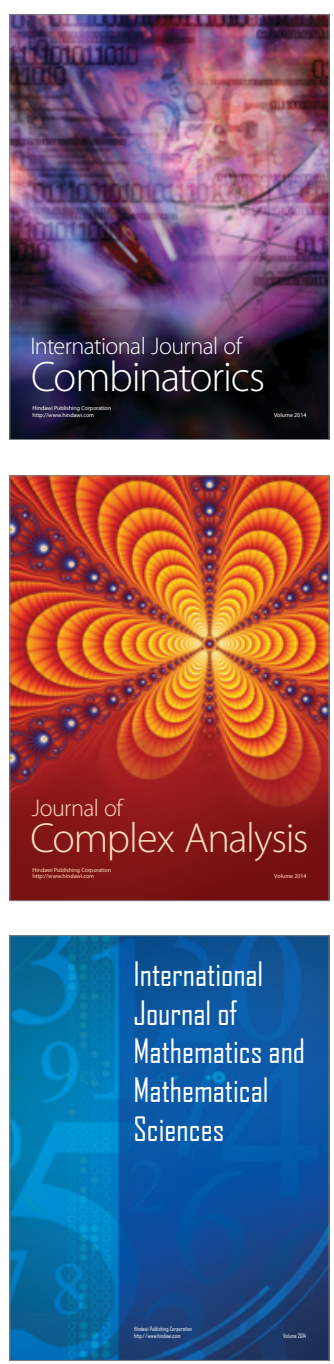
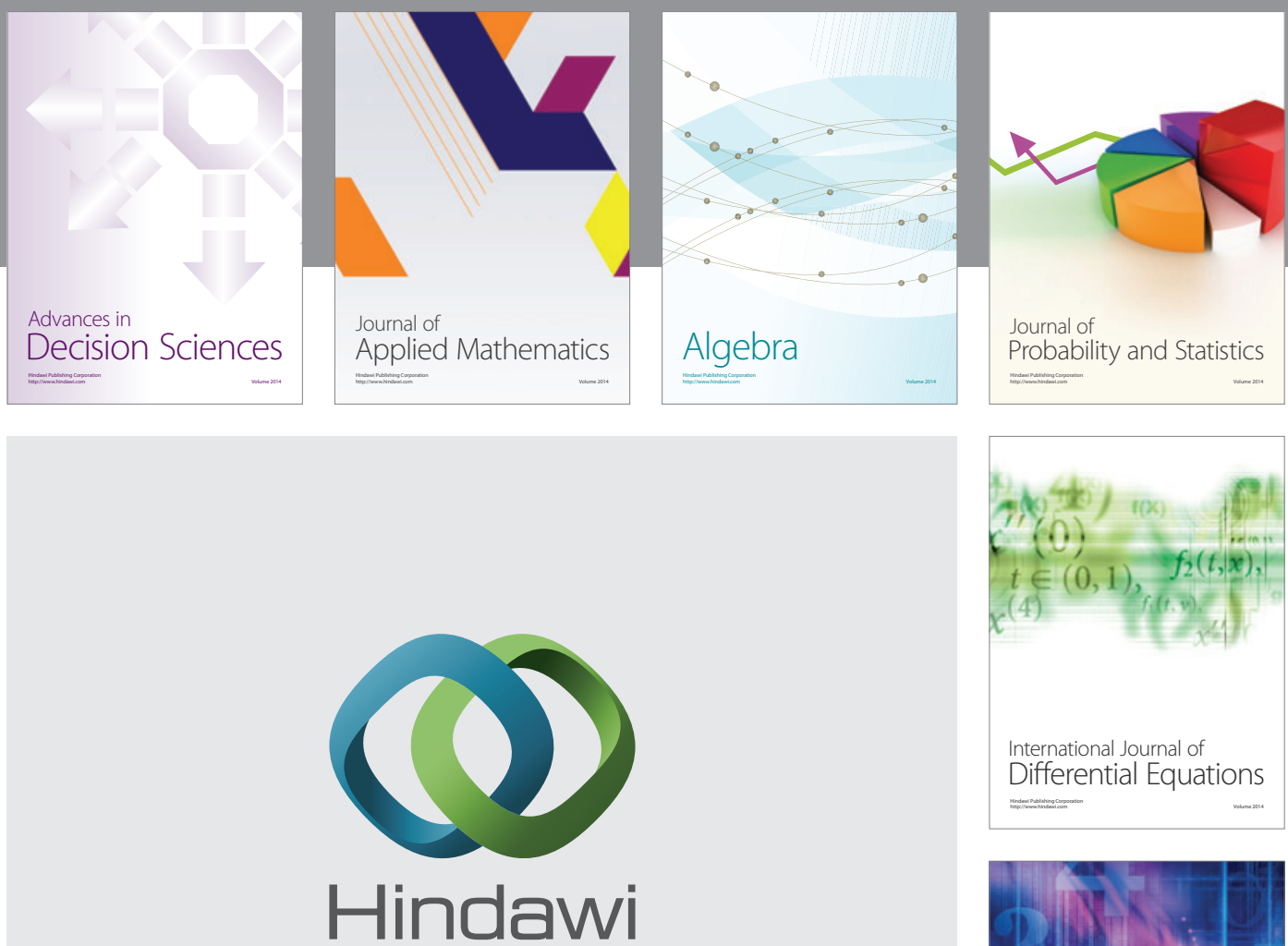

Submit your manuscripts at http://www.hindawi.com
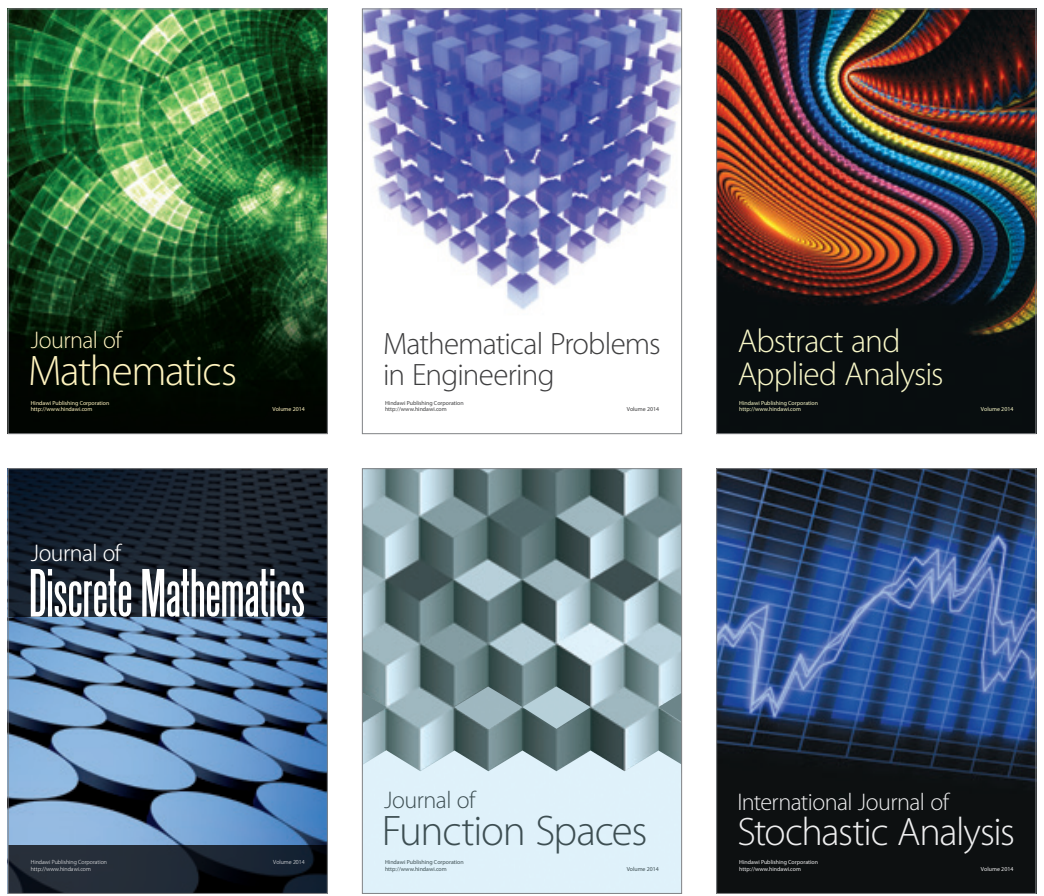

Journal of

Function Spaces

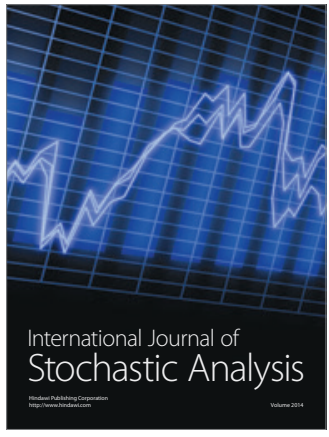

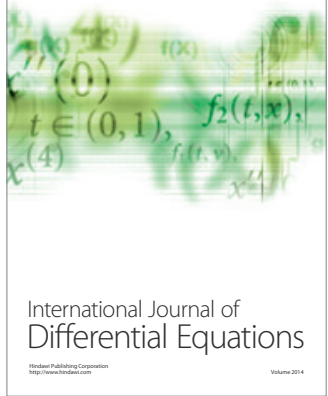
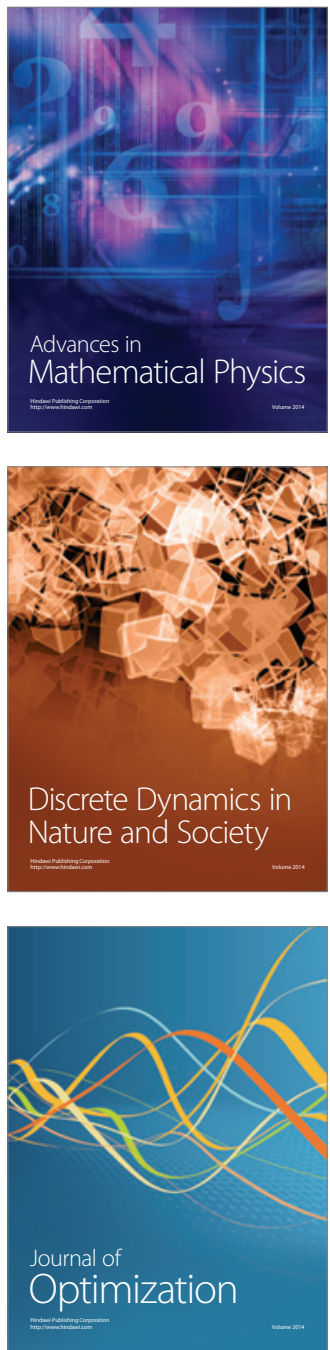